\title{
Análisis de la producción científica latinoamericana sobre rendimiento académico (2015-2018)
}

\section{Analysis of Latin American Scientific Production on Academic Performance (2015-2018)}

\author{
Dario Utrilla Salazar* \\ Universidad Nacional Mayor de San Marcos, Lima, Perú \\ ORCID: https://orcid.org/0000-0002-8098-3072 \\ Wilbert Chavez Irazabal \\ Universidad Nacional Mayor de San Marcos, Lima, Perú \\ ORCID: https://orcid.org/0000-0002-7978-7031 \\ Luz Marina Sito Justiniano \\ Universidad Nacional de Educación Enrique Guzmán y Valle, Lima, Perú \\ ORCID: https://orcid.org/0000-0001-6212-5982 \\ Guillermo Vargas Quispe \\ Universidad Nacional de Educación Enrique Guzmán y Valle, Lima, Perú \\ ORCID: https://orcid.org/0000-0002-0473-4146 \\ Aldo Rafael Medina Gamero \\ Universidad Continental, Lima, Perú \\ ORCID: https://orcid.org/0000-0003-3352-8779 \\ Melissa Rivera-Carrillo \\ Universidad César Vallejo, Lima, Perú \\ ORCID: https://orcid.org/0000-0003-2306-3912 \\ Ana María Vilchez Huerto \\ Universidad Nacional Mayor de San Marcos, Lima, Perú \\ ORCID: https://orcid.org/0000-0003-4666-3830
}

Recibido 20-10-19 Revisado 12-11-19 Aprobado 12-01-20 En línea 06-02-20

*Correspondencia

Email: dutrillas@unmsm.edu.pe
Citar como:

\footnotetext{
Utrilla, D., Chavez, W., Sito, L., Vargas, G., Medina, A., Rivera-Castillo, M., Vilchez, A. (2020). Análisis de la producción científica latinoamericana sobre rendimiento académico (2015-2018), 8(1), e452. doi: http://dx.doi.org/10.20511/pyr2020.v8n1.452
} 


\section{Resumen}

El estudio describe las características de producción científica latinoamericana sobre la variable rendimiento escolar en revistas indizadas en Scopus, durante el periodo 20152018. Se realiza un estudio descriptivo retrospectivo analizando 850 artículos. Se encuentra que los países que mayor producción tienen son Brasil y Chile. La mayoría de los artículos se encuentran publicados en las revistas Formación Universitaria y Psicología Escolar e Educacional. Se concluye que la producción científica latinoamericana sobre rendimiento académico ha ido aumentando progresivamente durante los últimos años; sin embrago, aún es necesario seguir fortaleciendo la generación de difusión de estudios científicos, además se recomienda utilizar técnicas de metaanálisis para llegar a conclusiones más integradoras que permitan seguir construyendo un conocimiento teórico.

Palabras clave: Rendimiento académico; Producción latinoamericana; Investigación; Educación.

\section{Summary}

The study describes the characteristics of Latin American scientific production on the variable school performance in journals indexed in Scopus, during the period 2015-2018. A retrospective descriptive study is carried out analyzing 850 articles. It is found that the countries with the highest production are Brazil and Chile. Most of the articles are published in the journals University Training and School and Educational Psychology. It is concluded that Latin American scientific production on academic performance has been increasing progressively in recent years; However, it is still necessary to continue strengthening the dissemination of scientific studies, it is also recommended to use meta-analysis techniques to reach more integrative conclusions that allow us to continue building theoretical knowledge.

Keywords: Academic performance; Latin American production; Investigation; Education.

\section{Introducción}

Uno de los ámbitos educativos y psicológicos que ha suscitado mayor interés en los investigadores, ha sido el relacionado con el rendimiento académico (Martín \& Serrano, 1994) y es que Edel (2003) menciona que esta variable una de las dimensiones más importantes en el proceso de enseñanza aprendizaje.

La complejidad del rendimiento académico inicia desde su conceptualización, en ocasiones se le denomina aptitud escolar, desempeño académico ó rendimiento escolar, pero generalmente las diferencias de concepto sólo se explican por cuestiones semánticas, ya que generalmente, en los textos la vida escolar y la experiencia docente, son utilizadas como sinónimos (Edel, 2003). El rendimiento académico puede ser conceptualizado como la valoración cuantitativa y cualitativa del logro de competencia alcanzado en el proceso de enseñanzaaprendizaje (Cardona, Vélez \& Tobón, 2016; García, 2018).

Arribas (2012), indica que el rendimiento académico se encuentra en función del sistema de evaluación y calificación empleado en el ámbito universitario. Los hallazgos confirman que la evaluación continua es la que propicia los mejores resultados en cuanto a la tasa de rendimiento y a las calificaciones obtenidas. Para Lamas (2015) el rendimiento académico se debe usar en poblaciones universitarias y rendimiento escolar en poblaciones de educación básica regular y alternativa (Fajardo Bullón, Maestre Campos, Felipe Castaño, León del Barco, \& Polo del Río, 2017). 
Lo anterior, nos hace preguntarnos por el avance en la investigación sobre rendimiento académico siendo necesaria la realización de este pre-estudio que tuvo como objetivo identificar el estado actual de la producción científica de esta variable en Latinoamérica, a partir de la revisión de los artículos publicados y disponibles en Scopus.

\section{Método}

Se analizaron 850 artículos que reportaban resultados de investigaciones realizadas sobre rendimiento académico escrita por autores con afiliación a instituciones latinoamericanas y que se encontraron en la base de datos Scopus, se escogió ésta por ser una de las más grande y reconocida de resúmenes y referencias bibliográficas de literatura científica y abarcó el periodo 2015-2018. Se utilizó una matriz de registro documental en base a la revisión detallada de cada uno de los artículos, en la que se registró: autores, título del artículo, año de publicación, idioma(s), nombre, URL y/o DOI de publicación.

Este estudio es de tipo descriptivo a partir de la revisión de documentos. Inicialmente se realizó la búsqueda de artículos utilizando palabras clave como: Academic achievement; Academic performance; Student achievement; Student progress; Achievement tests; Student evaluation; Educational rating; Talent identification; Student appraisal; Educational assessment; Educational testing; se exploró en los títulos/resúmenes/palabras clave, con el fin de recoger todas las opciones posibles.

\section{Resultados}

Con respecto al número de publicaciones por año, se observa un aumento de investigaciones gradual, evidenciando un incremento mayor en el $2018(n=263)$, ver figura 1.

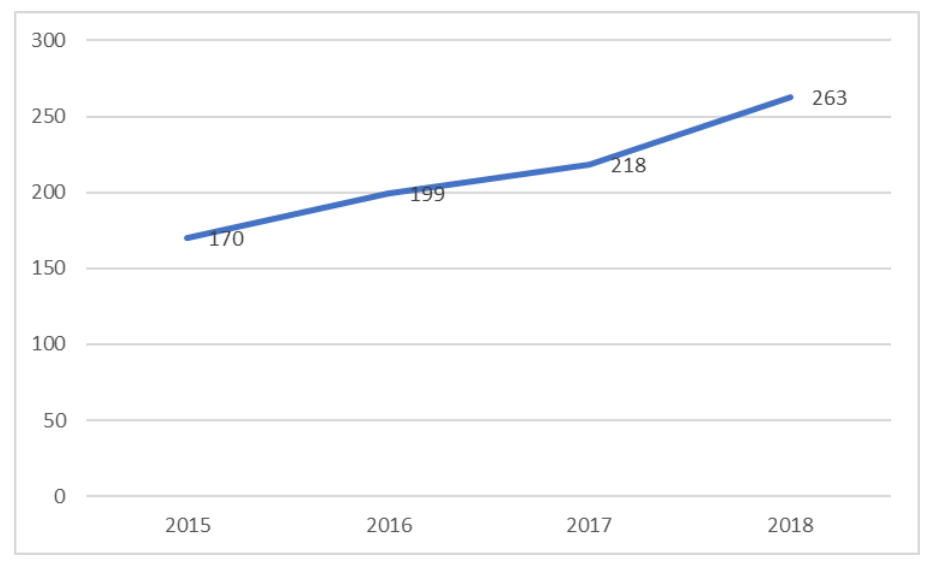

Figura 1. Histórico de producción científica sobre rendimiento académico en Scopus (2015-2018)

Los artículos analizados fueron publicados en 121 revistas. La tabla 1 muestra las revistas donde se han publicado mayoritariamente los artículos con referencia a la variable rendimiento académico. Formación Universitaria y Psicologia Escolar e Educacional son las revistas donde encontramos mayor cantidad de publicaciones sobre la variable estudiada, cabe mencionar que ambas son editadas por universidades latinoamericanas. 


\section{Tabla 1.}

Revistas indizadas en Scopus donde se ha publicado artículos referentes a rendimiento escolar (2015-2018)

\begin{tabular}{lccc}
\hline Revista & n & País & índice H \\
\hline Formación Universitaria & 18 & Chile & 10 \\
Psicologia Escolar E Educacional & 17 & Braasil & 6 \\
Espacios & 16 & Venezuela & 12 \\
Frontiers In Psychology & 16 & Suiza & 81 \\
Revista Cubana De Educacion Medica & 12 & Cuba & 11 \\
Superior & & & \\
Universitas Psychologica & 11 & Colombia & 18 \\
Codas & 10 & Braasil & 19 \\
Educacao E Pesquisa & 9 & Brasil & 13 \\
Plos One & 9 & Estados & 268 \\
& & Unidos & \\
Revista Electronica De Investigacion & 9 & México & 9 \\
Educativa & & & \\
Education Policy Analysis Archives & 8 & Estados & 40 \\
& & Unidos & \\
Ensaio & 8 & Brasil & 8 \\
IEEE Latin America Transactions & 8 & Estados & 19 \\
& & Unidos & \\
Interdisciplinaria & 8 & Argentina & 8 \\
Otras & & & \\
\hline
\end{tabular}

En relación con la contribución por país, se observa que Brasil tiene la mayor cantidad de autoría ( $\mathrm{n}=292)$, seguido de Chile $(\mathrm{n}=186)$, Colombia $(\mathrm{n}=120)$ y México $(\mathrm{n}=118)$ entre otros (ver tabla 2).

Tabla 2.

Afiliación institucional por país según publicaciones sobre rendimiento escolar en Scopus. (20152018)

\begin{tabular}{lc}
\hline País & n \\
\hline Brasil & 292 \\
Chile & 186 \\
Colombia & 120 \\
México & 118 \\
Argentina & 55 \\
Ecuador & 34 \\
Perú & 34 \\
Otros países & 11 \\
\hline
\end{tabular}

\section{Discusión}

Los hallazgos indican que durante los últimos cinco años las investigaciones sobre rendimiento escolar han aumentado, siendo el 2018 un año en el cual se destaca una mayor producción a nivel Latinoamericano, aparece Brasil y Chile como los países que mayor reconocimiento tiene en cuanto a los estudios que difunde. Perez y Nieto (1992) encontraron que el 50\% de los trabajos 
realizados sobre rendimiento académico en España no se publican en revistas especializadas, aunque existe un alto porcentaje que no se publica como en el caso de tesis o tesinas.

Un dato interesante y significativo es que en el estudio de Perez y Nieto (1992) la revista que aporta mayor porcentaje sobre rendimiento académico es la Revista de Investigación Educativa, de procedencia española, de igual forma nuestro estudio encuentra que las principales revistas de producción son latinoamericanas, concluyendo que el autor o grupo de autores buscan publicar sus estudios dentro de revistas locales o de su propio entorno (Pulido Acosta, \& Herrera Clavero, 2017).

El presente estudio como en todos aquellos que buscan determinar el alcance de producción de una variable se considera como un instrumento básico para conocer el estado de publicación; sin embargo, sería muy conveniente seguir utilizando técnicas de metaanálisis para llegar a conclusiones más integradoras que permitan seguir construyendo un conocimiento teórico.

\section{Referencias}

Arribas, J. (2012). El rendimiento académico en función del sistema de evaluación empleado. Revista Electrónica de Investigación y Evaluación Educativa, 18(1), 1-15. Recuperado de: http://www.uv.es/RELIEVE/v18n1/RELIEVEv18n1_3.htm

Cardona, S., Vélez, J., \& Tobón, S. (2016). Contribución de la evaluación socioformativa al rendimiento académico en pregrado. Educar, 52(2), 423-47. Doi: https://doi.org/10.5565/rev/educar.763

Edel, R. (2003). El rendimiento académico: concepto, investigación y desarrollo. REICE. Revista Iberoamericana sobre Calidad, Eficacia y Cambio en Educación, 1(2), 0.

Fajardo Bullón, F., Maestre Campos, M., Felipe Castaño, E., León del Barco, B., \& Polo del Río, M. I. (2017). Análisis del rendimiento académico de los alumnos de Educación Secundaria Obligatoria según las variables familiares. Educación XXI: revista de la Facultad de Educación, 20(1), 209-232.

García, A. E. (2018). Estilos de aprendizaje y rendimiento académico. Revista Boletín Redipe, 7(7), 218-228.

Lamas, H. A. (2015). Sobre el rendimiento escolar. Propósitos y representaciones, 3(1), 313-386. Doi: http://dx.doi.org/10.20511/pyr2015.v3n1.74

Martín, S., \& Serrano, G. (1994). Estudios e investigaciones sobre rendimiento académico (19701990): análisis estadístico y bibliométrico. Revista Española e Pedagogía, 52(199), 501527. Recuperado de www.jstor.org/stable/23764250

Perez, G., \& Nieto, S., (1992). Análisis estadístico y bibliométrico sobres investigaciones y estudios acerca del rendimiento académico en España (1976-1986): Aspectos conceptuales y descriptivos. Aula abierta, 60, 47-65. Recuperado de https://www.unioviedo.es/reunido/index.php/AA/issue/viewIssue/1019/95

Pulido Acosta, F., \& Herrera Clavero, F. (2017). La influencia de las emociones sobre el rendimiento académico. Ciencias Psicológicas, 11(1), 29-39. 